\title{
Implicit large eddy simulation of unsteady cloud cavitation around a plane-convex hydrofoil
}

\author{
Victor Hidalgo ${ }^{1}$, Xianwu Luo ${ }^{1 *}$, Xavier Escaler ${ }^{2}$, Bin $\mathrm{Ji}^{3}$, Alvaro Aguinaga ${ }^{4}$ \\ ${ }^{1}$ State Key Laboratory of Hydroscience and Engineering, Tsinghua University, \\ Beijing 100084, China \\ ${ }^{2}$ CDIF, Universitat Politècnica de Catalunya, \\ Barcelona, Spain \\ ${ }^{3}$ School of Power and Mechanical Engineering, Wuhan University, \\ Hubei 430072, China \\ ${ }^{4}$ Mechanical Engineering Department, Escuela Politecnica Nacional University, \\ Quito E11-253, Ecuador \\ *Correspondence author: luoxw@mail.tsinghua.edu.cn
}

\begin{abstract}
The present research, focuses on the erosive cavitation behavior around a plane convex hydrofoil. For that, Tthe Zwart-Gerber-Belamri cavitation model has been implemented in library form to be used withto earry out in OpenFOAM. Implicit Large Eddy Simulation (ILES) has been applied to analyze the three dimensional unsteady cavitating flow around a plane convexthe hydrofoil. The numerical results corresponding to hydrodynamic conditions that have been experimentally tested at the high speed cavitation tunnel of the École Polytechnique Fédérale de Lausanne (EPFL) show the sheet cavitation development and the shedding and collapse of vapor clouds. It is noted that cavitation evolution including maximum vapor length, detachment and oscillation frequency are fairly well simulated. Furthermore, the pressure pulses due to the cavitation development as well as complex vortex structures are reasonably predicted. Consequently, these results confirm that the present numerical method can be used to investigate unsteady cavitation around hydrofoils with satisfactory accuracy.
\end{abstract}

Formatat: Color de la lletra: Automàtic 


\section{Introduction}

Studies of unsteady cloud cavitation around hydrofoils are important to improve the design theory of fluid machinery $[1,2]$ and to understand the mechanisms, due to questions around the cavitation dynamic behavior and the collapse of the cloud of bubbles. However, experimental studies are expensive and have limitations such as the accuracy of lab equipments [3]. Therefore, computational fluid mechanics (CFD) are a complementary option to understand the cavity shedding and collapse, the cyclic stresses being generated and the resulting cavitation erosion.

In this way, Kunz et al. [4] proposed a cavitation model based on Merkle's ideas for the analysis of unsteady cavitating flow. The model considers the continuity of a mixture volume rather than a mixture mass equation. The transfer from liquid to vapor is modeled as being proportional to the liquid volume fraction and the pressure below the vapor pressure. For instance, Nouri et al. [5] studied unsteady cavitating flow over a disc using OpenFOAM with explicit LES based on this cavitation model. Their results showed that the main aspects of super cavitation could be captured. However, selecting the correct explicit subgrid model is shown to be a problem for different cases. Moreover, the shape of the cluster of bubbles can be improved with a cavitation model based on Rayleigh-Plesset's equation. Based on this premise, Roohi et al. [6] studied the cavitating flow over the Clark-Y hydrofoil and they compared the Kunz model with Schnerr-Sauer cavitation model [7] which is based on Rayleigh-Plesset's equation and is part of the default OpenFOAM solver packages too. The corresponding results showed that the Schnerr-Sauer model predicted a stronger re-entrant jet, which was visible in experiment, and the shape of the obtained cavity was improved as well. However, it was found that the symmetrical consideration for condensation and vaporization processes may induce the high error of the force coefficients for super cavitation regime.

Consequently, Zwart et al. [8] presented a two phase flow model for predicting cavitation dynamics which was implemented in CFX-5 based on Rayleigh-Plesset's equation. The model worked well for condensation but it was unstable for vaporization. So, such unsymmetrical consideration was taken into account with empirical constants for calibration. As a result, the model shows improvements for non-equilibrium effects, which were not included in previous models as Schnerr- Sauer, Gerber [9], Senocak and Shyy [10]. Different researchers have used this model to capture the cavitation phenomenon with good results. That is the case of Ji et al. [11, 12] who studied the horse-shoe cloud behavior over a twisted hydrofoil and predicted the performances of a marine propeller under cavitation condition with different skew angles. Shi et al. [13] also showed the remarkable use of the Zwart-Gerber-Belamri model for the study of cavitation in a WP7 automobile centrifugal pump. Zhang et al. [14] used this model to study the effects of density ratio on the maximum length of the attached sheet of vapor. Some studies have been carried out to improve the model as the case of Morgut et al. [15] who determined the possible values of the calibration constants for numerical simulations. In this line, Li et al. [16] studied the influences of the empirical constants and the nucleation

| volume fraction for the model. From their research, it was concluded that the evaporation coefficient controls the cavity length and the high vapor volume fraction, while the condensation vapor regulates the cavity length. Unfortunately, the cavitation model of ZwartGerber-Belamri is not part of the OpenFOAM solvers and no references are available of

Formatat: Color de la lletra: 
previous works with this solver. Therefore, its proper implementation and validation is a challenge, which is the main objective of the current work.

Due to the high Reynolds numbers of cavitation flows in hydro machinery, the traditional RANS and turbulence models must be adapted to predict the cavity sheet and the shedding process. Some examples are the cases of Huang and Wang [17] and Ji et al. [18] who used Partially-Averaged Navier-Stokes (PANS) equations in the commercial code ANSYS-CFX for the cavitation simulation. PANS is a hybrid turbulence model which conducts the simulation from RANS to Direct Numerical Simulation (DNS) based on unresolved to total ratios of kinetic energy $\left(\mathrm{f}_{k}\right)$ and dissipation $\left(\mathrm{f}_{\varepsilon}\right)$. Nevertheless, those ratios can only be found by a subgrid independence analysis and they can not be easily estimated according to Sharath and Girimaji [19]. Another solution was proposed by Zuo et al. [20] who added a function to the $\mathrm{k}-\varepsilon$ turbulence model to consider the influence of variations in density on the turbulence viscosity for the study of cavitation in a Francis turbine. Looking for a better solution, Bensow and Bark [21] and Lu et al. [22] worked with OpenFOAM to use large eddy simulation (LES) instead of RANS to simulate the formation of erosive cavitation based on Implicit Large Eddy Simulation (ILES). ILES was selected to avoid the explicit coupling between mass transfer modeling and subgrid modeling. ILES has been validated by recent studies on aerospace and naval engineering for highly turbulent flows such as the flows over a fully three-dimensional swept-wing geometry [23] and a marine propeller [24].

In summary, the main contribution in this paper will be the implementation and validation of the Zwart-Gerber-Belamri cavitation model in OpenFOAM using ILES. Therefore, it is intended to obtain accurate simulations of the shedding process of partial cavitation, which is considered the main mechanism of material erosion. For validation purposes, the experimental results obtained by Escaler et al. [25, 26] with a 2D plane-convex hydrofoil at the École Polytechnique Fédérale de Lausanne (EPFL) high speed cavitation tunnel have been used. Previous comparisons with OpenFOAM were focused on NACA series or twisted geometries with unsteady cavitation [27, 28, 29] to compare the cavitation hydrodynamic behavior. For the present paper, the plane-convex hydrofoil was specifically investigated to reveal the unsteady cavitation dynamics.

\section{Description of the numerical method}

\subsection{Implicit Large Eddy Simulation}

Continuity and Navier-Stokes equations are the basis of LES, as shown in Eqs. 1 and 2.

$$
\begin{gathered}
\frac{\partial \rho}{\partial t}+\frac{\partial\left(\rho u_{i}\right)}{\partial x_{i}}=0 \\
\frac{\partial}{\partial t}\left(\rho u_{i}\right)+\frac{\partial}{\partial x_{j}}\left(\rho u_{i} u_{j}\right)=-\frac{\partial p}{\partial x_{i}}+\frac{\partial}{\partial x_{j}}\left[\rho v\left(\frac{\partial u_{i}}{\partial x_{j}}+\frac{\partial u_{j}}{\partial x_{i}}\right)_{-}^{-}\right.
\end{gathered}
$$

where $u$ is the instant velocity, $t$ is time, $i$ and $j$ are the space axes subscripts.

In ILES, Eqs. 1 and 2 are filtered to use the approximation- and to exclude some solutions, which are not relevant for Eqs. 3 and 4 [30].

Formatat: Color de la lletra:

Formatat: Color de la lletra: 


$$
\begin{gathered}
\frac{\partial \rho}{\partial t}+\frac{\partial\left(\rho \overline{u_{i}}\right)}{\partial x_{i}}=0 \\
\frac{\partial}{\partial t}\left(\rho \overline{u_{i}}\right)+\frac{\partial}{\partial x_{j}}\left(\rho \overline{u_{i} u_{j}}\right)=-\frac{\partial \bar{p}}{\partial x_{i}}+\frac{\partial}{\partial x_{j}}\left[\rho v\left(\frac{\partial \overline{u_{i}}}{\partial x_{j}}+\frac{\partial \overline{u_{j}}}{\partial x_{i}}\right)\right] .
\end{gathered}
$$

Then Eq. 4 is reduced to obtain Eq. 5 by the following considerations:

1. The product of filtered velocities is $\overline{u_{i} u_{j}}=\bar{u}_{i} \bar{u}_{j}+\overline{u_{i}^{\prime} u_{j}^{\prime}}$.

2. The subgrid stress tensor, which is the Reynolds stress tensor is $\tau_{i j}^{\prime}=\rho \overline{u_{i}^{\prime} u_{j}^{\prime}}=\rho\left(\overline{u_{i} u_{j}}-\bar{u}_{i} \bar{u}_{j}\right)$.

3. The filtered strain tensor rate is $\bar{S}_{i j}=\frac{1}{2}\left(\frac{\partial \bar{u}_{i}}{\partial x_{j}}+\frac{\partial \bar{u}_{j}}{\partial x_{i}}\right)$.

4. The filtered viscous stress tensor is $\bar{\tau}_{i j}=2 \rho v \bar{S}_{i j}$.

$$
\frac{\partial\left(\rho \overline{u_{i}}\right)}{\partial t}+\frac{\partial\left(\rho \bar{u}_{i} \bar{u}_{j}\right)}{\partial x_{j}}=-\frac{\partial \bar{p}}{\partial x_{i}}+\frac{\partial\left(\bar{\tau}_{i j}-\tau_{i j}^{\prime}\right)}{\partial x_{j}} .
$$

The subgrid stress tensor $\tau_{i j}^{\prime}$ is a nonlinear term, so it is separated as indicated in Eq. 6.

$$
\tau_{i j}^{\prime}=\rho\left(\overline{\bar{u}}_{i} \bar{u}_{j}-\overline{\bar{u}}_{i} \overline{\bar{u}}_{j}+\tilde{\tau}_{i j}^{\prime}\right)
$$

where, the tensor $\tilde{\tau}_{i j}^{\prime}$ according to Bensow-Bark [21] and Lu et al. [22] is modeled by using the truncation error of the discretization, which acts as a dissipative action to obtain an implicit subgrid scale (SGS) for ILES. Adams et al. [30] gives more details about the mathematical implementation of this implicit model.

\subsection{Zwart-Gerber-Belamri cavitation model}

The cavitating flow is considered as a single fluid with two-phase homogeneous mixture with phase changes between vapor and water. Eq. 7 to 9 indicate the vapor volume fraction $\alpha$, the

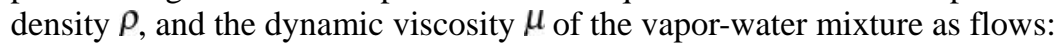

$$
\begin{gathered}
\alpha=\frac{V_{v}}{V}, \\
\rho=(1-\alpha) \rho_{l}+\alpha \rho_{v}, \\
\mu=(1-\alpha) \mu_{l}+\alpha \mu_{v},
\end{gathered}
$$

where $V$ is the total volume, $l$ and $v$ are subscripts for liquid and vapor respectively. Thus Eq. 10 available based on Eq. 3, which considers the phase transfer called as the transport equation.

$$
\frac{\partial\left(\alpha \rho_{v}\right)}{\partial t}+\frac{\partial\left(\alpha \rho_{v} \bar{u}_{i}\right)}{\partial x_{i}}=\dot{m}
$$

where $\dot{m}$ is the inter-phase mass transfer rate per unit volume.

The Zwart-Gerber-Belamri model considers a variable bubble radius, $R$, based on Eq. 11, in which the second derivative term is neglected to simplify the expression and to get Eq. 12. 


$$
\begin{aligned}
& R \frac{\partial^{2} R}{\partial t^{2}}+\frac{3}{2}\left(\frac{\partial R}{\partial t}\right)^{2}=\frac{p-p_{v}}{\rho_{l}} \\
& \frac{d R}{d t}=\sqrt{\frac{2}{3}\left(\frac{\left|p-p_{v}\right|}{\rho_{l}}\right)} .
\end{aligned}
$$

Thus the Zwart-Gerber-Belamri model is expressed with Eq. 13.

$$
\dot{m}=\left\{\begin{array}{ll}
\dot{m}^{+}=F_{V} \frac{3 r_{\text {nuc }}(1-\alpha) \rho_{v}}{R_{B}} \sqrt{\frac{2}{3}\left(\frac{p_{v}-p}{\rho_{l}}\right)} & \text { if } p<p_{v} \\
\dot{m}^{-}=-F_{\mathrm{C}} \frac{3 \alpha \rho_{v}}{R_{B}} \sqrt{\frac{2}{3}\left(\frac{p-p_{v}}{\rho_{l}}\right)} & \text { if } p>p_{v}
\end{array} .\right.
$$

where $F_{V}=300$ and $F_{C}=0.03$ are the selected calibration constants for vaporization and condensation, $r_{n u c}=5.0 \times 10^{-6}$ is the nucleation site volume fraction and $R_{B}=1.0 \times 10^{-6} \mathrm{~m}$ is the typical bubble size in water [29]. The work of Morgout et al. [15] has been used to choose $F_{V}$ and $F_{C}$ values. Moreover, a preliminary study of $r_{n u c}$ sensitivity was carried out to select its value.

The Zwart-Gerber-Belamri model has been written in C++ based on the OpenFOAM code. The code has been saved on the new solver directory and compiled to become an implemented OpenFOAM's cavitation model for the present research.

\subsection{OpenFOAM set up}

The OpenFOAM version 2.2.x was used in the present research because it solves the bugs of the previous version [17]. GNU/Linux Mint 6 Petra with kernel Linux 3.11.0-12-generic (x86 64) and XFCE desktop were also used. This version of OpenFOAM is used especially in the CentOS GNU/Linux distribution, which is based on the official GNU/Linux version of RedHAT. In fact, the following implementations were carried out:

1. In the directory OpenFOAM/OpenFOAM-2.2.x/etc the text document bashrc was modified. The lines "foamCompiler=system" and "WMCOMPILER=Gcc" were written and the line "foamCompiler=ThirdParty" was eliminated in the compiler location (\#Compiler Location:). These actions had to be made to compile the implemented cavitation solver of Zwart-Gerber-Belamri [29].

2. The library libmpi.so.1 is required in GNU/Linux Mint Petra. Thus, a symbolic link (ln s) with libmpi.so was implemented, which is part of Message Passing Interface (MPI) GNU/Linux library [31].

\section{Hydrofoil geometry, mesh generation and boundary conditions}

\subsection{Hydrofoil geometry and computation domain}

Figure 1 shows a plane-convex hydrofoil used in Escaler's studies [25, 26]. This hydrofoil presents a plane upper surface, a convex lower surface. The hydrofoil has a chord length c equal to $91.1 \mathrm{~mm}$, a span-length equal to $0.3 \mathrm{c}$, a semicircular leading edge and a tip trailing edge. 
The hydrofoil is positioned in a computation domain with an angle of attack, $\widehat{A O A}$, as shown in Fig. 2.

\subsection{Mesh generation}

Meshing is a challenge for this type of plane-convex hydrofoils due to the fact that the leading edge presents a great change from a convex shape to a plane shape with a semicircular leading edge.

Therefore, a structured C mesh generated using Gmsh 2.8.5 and Salome 7.4.0 was obtained with scale distribution and hexahedron elements. The resulting mesh has 393980 hexahedra. The quality of the mesh is guaranteed by the Salome taper analysis as indicated in Fig. 3. This analysis shows proportional changes of elements such as 0.021 , which is an acceptable value according to previous works [28].

The Yplus, $y^{+}$, is calculated based on Eq. 14 and the mean value for the hydrofoil wall is 9.2. This mean value is within the range from 1 to 15 , which ensures that the mesh matches the conditions for ILES based on Lu et al. [22].

$$
y^{+}=\frac{u_{\tau} y}{v}
$$

where $u_{\tau}$ is the friction velocity, $y$ is the distance to the nearest hydrofoil wall and $v$ is the kinematic viscosity [29].

\subsection{Boundary Conditions}

Both, the Reynolds number, $R e$, and the cavitation number, $\sigma$, indicated in Eqs. 15 and 16 are applied to show the cavitating flow conditions:

$$
\begin{aligned}
& R e=\frac{U_{\infty} c}{v}: \\
& \sigma=\frac{p_{r}-p_{v}}{0.5 \rho U_{\infty}^{2}} .
\end{aligned}
$$

where $U_{\infty}$ is the free stream velocity [15, 32], $c$ is the chord length, and $p_{r}$ and $p_{v}$ are the reference and saturation pressures, respectively.

Based on the experimental tests carried out by Escaler [25], five flow conditions combining different free stream velocity at domain inlet, angle of attack and the reference pressure at domain outlet have been selected as shown in Tab. 1 . The uniform velocity and the static pressure were assassinated for the domain inlet and outlet.

Table 1: Simulated flow conditions

\begin{tabular}{ccccc}
\hline Cases & $\sigma$ & $\widehat{A O A}[\mathrm{o}]$ & $U_{\infty}[\mathrm{m} / \mathrm{s}]$ & $p_{r}[\mathrm{kPa}]$ \\
\hline (i) & 0.70 & 2.3 & 35.0 & 430.19 \\
(ii) & 0.70 & 3.0 & 25.0 & 220.60 \\
(iii) & 0.70 & 3.0 & 35.0 & 430.19 \\
(iv) & 1.00 & 3.0 & 35.0 & 613.58 \\
(v) & 1.12 & 3.0 & 35.0 & 680.82 \\
\hline
\end{tabular}


The front and back planes of the domain were considered as symmetry boundaries. Besides, the top and bottom of the computation domain, the hydrofoil surface were treated as no-slip wall.

\section{Results and Discussion}

Figure 4 shows several characteristic parameters defined for the cavity attached to the hydrofoil such as the cavity length $L$, the maximum cavity length $L_{\max }$, the depth $H_{\max }$, etc. The cavitating flow over the plane-convex hydrofoil presents a typical partial cavitation development consisting of an attached cavity from the leading edge. Under unsteady conditions, the re-entrant jet mechanism provokes the periodic shedding of bubble clouds [26].

Strouhal number, Str, is a typical dimensionless parameter to show the feature of cavity oscillation, as indicated in Eq. 17.

$$
S t r=\frac{f L_{\max }}{U_{\infty}},
$$

where $f$ is the cavity oscillation frequency and $U_{\infty}$ is the mean velocity of the free stream.

The transient numerical results have been post-processed and analyzed along the time to identify a single period of the cavitation oscillation process. Particular attention has been given to the vapor volume fraction, the development of the reentrant jet, the shedding of a cloud cavity and its final collapse. The cloud cavity like the horse-shoe is visible and there is a good similarity between the experiment and simulation, as indicated in Fig. 5.

For a better comparison, the dimensionless time given by Eq. 18 has been used.

$$
\xi=\frac{t-t_{o}}{t_{f}-t_{o}} .
$$

where $t$ is a time between initial time, $t_{o}$, and final time, ${ }_{f}$, in one cycle. In particular six time instants corresponding to $0,1 / 6,1 / 3,1 / 2,2 / 3$ and $5 / 6$ have been selected, and the corresponding contour of the vapor volume fraction $\alpha=0.5$ are plotted in Fig. 6, for all flow conditions.

Based on Fig. 6 the following information can be obtained:

1. All the cases show the growth and detachment of leading edge cavity, and the break off and collapse of the cavity cloud.

2. The maximum length of the leading edge attached cavity decreases with the increase of the cavitation number.

3. With $\sigma=0.7$, the cavity cloud is observed more clearly for case (i) and (iii) at $U_{\infty}=35$ $\mathrm{m} / \mathrm{s}$ than that for case (ii) at $U_{\infty}=25 \mathrm{~m} / \mathrm{s}$. Therefore, the larger velocity enhances the probabilities of cloud cavity, which is linked to erosive power of cavitation erosion.

4. Cavities at the hydrofoil trailing edge were observed along the lower surface of the hydrofoil for three cases with $\sigma=0.7$. This kind of cavitation is due to the hydrofoil convex shape and it is also observed experimentally. 
5. It is noted that results obtained by ILES method has good accuracy as previous studies [33] based on explicit subgrid model of LES.

In order to understand the dimensionless evolution of cavity shedding shown in Fig. 6, the pressure coefficient, $C p$, calculated based on Eq. 19 and the vapor void fraction, $\alpha$, have been plotted in Fig. 7 and Fig. 8, respectively, as a function of $\xi$ for six points located along the upper surface at $0.15 c$ in spanwise direction.

$$
C p=\frac{p_{s}-p_{r}}{0.5 \rho U_{\infty}^{2}}
$$

where $p_{s}$ is the static pressure.

According to the definition of $\sigma$ and $C p$, there is the following relation:

$$
C p=-\sigma+\frac{p_{s}-p_{v}}{0.5 \rho U_{\infty}^{2}} .
$$

Due to the fact that $p_{s}$ is always larger than $p_{v}, C p$ must be larger than $-\sigma$.

Because those cases with $\sigma=0.7$ have larger cavity lengths, the flat $C p$ lines, whose value corresponding to the cavitation number are observed at $x / c=0.2$ and 0.4 , compared with other two cases. On the contrary, the cavity length of case (v) is shortest, and the static pressure oscillation violently even at $x / c=0.2$. From $x / c=0.6$ to $1.0, C p$ lines with strong pressure oscillations can be observed for all cases. At the downstream of the hydrofoil $(x / c=$ 1.2) , the average static pressure is basically around the reference pressure level, and some pressure pulses can be captured. Those pulses may relates with the collapse of vapor droplet. Based on vapor volume fraction oscillations shown in Fig. 8, the vapor sizes are larger for case (i) and (iii), and the vapor size is smallest for case (v) due to its highest reference pressure.

The comparison of cavity length among those five cases is listed at Tab. 2, where both experimental and simulation data are included. To show the difference between the experiment and calculation, Eq. 21 defines the error evaluation of the relative cavity length. The prediction accuracy is fairly good, though the largest error is near $20 \%$. It must be noted that due to the transient nature of the shedding process and the high frequencies involved during the tests [25], the maximum length could not be determined with sufficient accuracy from the experiments so an error of about $\pm 5 \%$ can be assumed.Probably the error can decrease if the experiments can be repeated with the goals to measure the cavity length and frequency, which were not the main objects of EPFL's researchers in 2003 [25].

$$
\text { error }=\frac{\left|\left(L_{\max } / c\right)_{\text {Exp. }}-\left(L_{\max } / c\right)_{\text {Num. }}\right|}{\left(L_{\max } / c\right)_{\text {Exp. }}} \times 100 \%
$$

Table 2: Numerical studies and experimental results based on $L_{\max }$

\begin{tabular}{cccccc}
\hline & \multicolumn{4}{c}{$L_{\max } / c$} & $f$ \\
Cases & Num. & Exp. & Error [\%] & Num. [Hz] & Num. \\
\hline (i) & 0.47 & 0.50 & 6.0 & 166.67 & 0.20
\end{tabular}




$\begin{array}{cccccc}\text { (ii) } & 0.40 & 0.50 & 20.0 & 163.93 & 0.24 \\ \text { (iii) } & 0.44 & 0.45 & 2.2 & 151.51 & 0.18 \\ \text { (iv) } & 0.13 & 0.16 & 18.7 & 588.24 & 0.20 \\ \text { (v) } & 0.12 & 0.14 & 14.3 & 588.24 & 0.18\end{array}$

For a better understanding of the phenomenon, the Q-criterion based on Eq. 22 is used to obtain the 3D vorticity distribution.

$$
Q=\frac{1}{\Omega^{2}-S^{2}}
$$

where $\Omega$ is the vorticity rate, $S$ is the strain rate.

The $Q$-criterion is plotted in Fig. 9 where iso-contours of $Q=2 \times 10^{5} \mathrm{~s}^{-2}$ show similarities with vapor volume fraction iso-contours $(\alpha=0.5)$. Results depicted that there were strong interaction between the vorticity and cavitation evolution.

\section{Conclusions}

Formatat: Color de la lletra: Automàtic

A numerical simulation of unsteady cloud cavitation around a plane convex hydrofoil has been carried out with a free OpenFOAM software package using Implicit Large Eddy Simulation (ILES) where-and a newly implemented Zwart-Gerber-Belamri cavitation model were implemented. -The transient results at different operation conditions show the typical cavitation dynamic behaviors including the growth of the leading edge cavity, the development of the reentrant jet, the shedding of bubble clouds and their collapses.

The pressure oscillations due to cavitation evolution-dynamics as well as the complex vortex structures observed with $Q$-criterion were have been reasonably predicted. Further, a strouhal number between 0.18 and 0.24 denotes that the cavitation mechanics ean be reproducedare well captured. In conclusion, the proposed numerical method are-has been validated as a reliable tool for numerical studies and it can be used in future research to study cavitation-erosion mechanicsmechanisms.

\section{Acknowledgement}

This work was financially supported by the National Natural Science Foundation of China (Project Nos. 51306018, 51206087 and 51179091) and State key Laboratory of Hydroscience and Engineering, Tsinghua University (Project Nos. 2014-KY-05 and 2015-E-03).

\section{Conflict of interest}

Victor Hidalgo, Xianwu Luo, Xavier Escaler, Bin Ji and Alvaro Aguinaga declare that they have no conflict of interest.

\section{References and Notes}

[1] FRANC J.. and MICHEL J. Fundamentals of cavitation[M]. Dordrecht, The Netherlands: Springer, 2005, 1993-221. 
[2] LUO X., JI B. and XU H. Design and Optimization for Fluid Machinery[B]. Tsinghua University Press, 2012.

[3] ESCALER X., EGUSQUIZA E., FARHAT M., AVELLAN F., and COUSSIRAT M. Detection of cavitation in hydraulic turbines[J]. Mechanical systems and signal processing, 2006, 20(4):983-1007.

[4] KUNZ R., BOGER D., STINEBRING D., CHYCZEWSKI T., LINDAU J., GIBELING H., VENKATESWARAN S. and GOVINDAN T. A preconditioned Navier Stokes method for two-phase flows with application to cavitation prediction[J]. Computers \& Fluids, 2000, 29(8):849-875.

[5] NOURI N., MOGHIMI M., and MIRSAEEDI S. Numerical simulation of unsteady cavitating flow over a disc $[\mathrm{J}]$. Proceedings of the Institution of Mechanical Engineers, Part C: Journal of Mechanical Engineering Science, 2010, 224(6):1245-1253.

[6] ROOHI E., ZAHIRI A., and ANDIDEH-FARD M. Numerical simulation of cavitation around a two-dimensional hydrofoil using VOF method and LES turbulence model[J]. Applied Mathematical Modelling, 2013, 37(9):6469-6488.

[7] SCHNERR G. and SAUER J. Physical and numerical modeling of unsteady cavitation dynamics $[C]$. Proceedings of International Conference on Multiphase Flow ICMF, Orleans, USA, 2001.

[8] ZWART P., GERBER A. and BELAMRI T. A Two-Phase Flow Model for Predicting Cavitation Dynamics[C]. Proceedings of International Conference on Multiphase Flow ICMF 2004, Yokohama, Japan, 2004.

[9] GERBER A. A CFD model for devices operating under extensive cavitation conditions[C]. Proceedings of IMECE, New Orleans, Louisina, 2002.

[10] SENOCAK I. and SHYY W. Evaluation of cavitation models for navier-stokes computations[C]. Proceedings of FEDSM, Montreal, Quebec, Canada, 2002.

[11] JI B., LUO X., WU Y., PENG X. and DUAN Y. Numerical analysis of unsteady cavitating turbulent flow and shedding horse-shoe vortex structure around a twisted hydrofoil[J]. International Journal of Multiphase Flow, 2013, 51:33-43.

[12] JI B., LUO X. and WU Y. Unsteady cavitation characteristics and alleviation of pressure fluctuations around marine propellers with different skew angles[J]. Journal of Mechanical Science and Technology, 2014, 28(4):1339-1348.

[13] SHI W., WANG C., WANG W., and PEI B. Numerical Calculation on Cavitation Pressure Pulsation in Centrifugal Pump[J]. Advances in Mechanical Engineering, 2014, 6:367631.

[14] ZHANG G., SHI W., ZHOU L. and ZHANG D. Effect of the Maximun Density Ratio Between Liquid and Vapor on Cavitating Simulation[J]. American Journal of Engineering and Applied Sciences, 2015, 9(1): 119-126.

[15] MORGUT M. and NOBILE E. Influence of the mass transfer model on the numerical prediction of the cavitating flow around a marine propeller[C]. Proceding of The Second International Symposium on Marine Propulsors, Hamburg, Germany, 2011.

[16] LIU H., WANG J., WANG Y., ZHANG H. and HUANG H. Influence of the empirical coefficients of cavitation model on predicting cavitating flow in the centrifugal pump[J]. International Journal of Naval Architecture and Ocean Engineering, 2014, 6:119-131. 
[17] HUANG B. and WANG G. Partially Averaged Navier-Stokes method for timedependent turbulent cavitating flows[J]. Journal of Hydrodynamics, Ser. B, 2011, 23(1):2633.

[18] JI B., LUO X., WU Y., PENG X., and XU H. Partially-Averaged Navier Stokes method with modified $\mathrm{k}$ model for cavitating flow around a marine propeller in a non-uniform wake[J]. International Journal of Heat and Mass Transfer, 2012, 55(2324):6582-6588.

[19] GIRIMAJI S. and ABDOL-HAMID K. Partially-Averaged Navier Stokes Model for Turbulence: Implementation and Validation[C]. Proceeding of 43rd AIAA Aerospace Sciences Meeting and Exhibit, Reno, Nevada, 2005.

[20] ZUO Z., LIU S., LIU D., QIN D. and WU Y. Numerical Predictions of the Incipient and Developed Interblade Vortex Lines of a Model Francis Turbine by Cavitation Calculations[J]. Advances in Mechanical Engineering, 2013, 5:397583.

[21] BENSOW R. and BARK G. Simulating cavitating flows with LES in OpenFoam[C]. Proceeding of V European Conference on Computational Fluid Dynamics, Lisbon, Portugal, 2010.

[22] LU N., BENSOW R. and BARK G. LES of unsteady cavitation on the Delft twisted foil[J]. Journal of Hydrodynamics, Ser. B, 2010, 22(5, Supplement 1):784-791.

[23] HAHN M. and DRIKAKIS D. Implicit Large-Eddy Simulation of Swept-Wing Flow Using High-Resolution Methods[J]. AIAA Journal, 2009, 47(3):618-630.

[24] BENSOW R. and LIEFVENDAHL M. Implicit and explicit subgrid modeling in LES applied to a marine propeller[C]. Proceeding of 38th Fluid Dynamics Conference and Exhibit, Seattle, Washington, 2008.

[25] ESCALER X., FARHAT M., AVELLAN F. and EGUSQUIZA E. Cavitation erosion tests on a 2d hydrofoil using surface-mounted obstacles[J]. Wear, 2003, 254(5):441-449.

[26] ESCALER X., FARHAT M., EGUSQUIZA E. and AVELLAN F. Dynamics and Intensity of Erosive Partial Cavitation[J]. Journal of Fluids Engineering-transactions of The ASME, 2007, 129(7).

[27] HUUVA T., CURE A., BARK G. and NILSSON H. Computations of unsteady cavitating flow on wing profiles using a volume fraction method and mass transfer models[C].

Proceeding of 2nd IAHR International Meeting of the Workgroup on Cavitation and Dynamic Problems in Hydraulic Machinery and Systems, Timisoara, Romania, 2007.

[28] HIDALGO V., LUO X., JI B., and AGUINAGA A. Numerical study of unsteady cavitation on 2D NACA0015 hydrofoil using free/open source software[J]. Chinese Science Bulletin, 2014, 59(26):3276-3282.

[29] HIDALGO V., LUO X., ESCALER X., JI J. and AGUINAGA A. Numerical investigation of unsteady cavitation around a NACA 66 hydrofoil using OpenFOAM[J]. IOP Conference Series: Earth and Environmental Science, 2014, 22(5):052013.

[30] ADAMS N., HICKEL S. and FRANZ S. Implicit subgrid-scale modeling by adaptive deconvolution[J]. Journal of Computational Physics, 2004, 200(2):412-431.

[31] HIDALGO V., LUO X., HUANG R.and CANDO E. Numerical simulation of cavitating flow over 2D hydrofoil using openFOAM adapted for Debian operating system with LXDE based in kernel GNU/Linux[C]. Proceedings of the ASME 2014 4th Joint USEuropean Fluids Engineering, Chicago, Illinois, US, 2014. 
[32] VALLIER A., NILSSON H. and REVSTEDT J. Mass transfer cavitation model with variable density of nuclei[C]. Proceeding of 7th International Conference on Multiphase Flow, Tampa, Florida, 2010.

[33] JI B., LUO X., PENG X. and WU Y. Three-dimensional large eddy simulation and vorticity analysis of unsteady cavitating flow around a twisted hydrofoil[J]. Journal of Hydrodynamics, Ser. B, 2013, 25(4):510-519.

[34] PETKOVSEK M. and DULAR M. Simultaneous observation of cavitation structures and cavitation erosion[J]. Wear, 2013, 300(2013):55-64. 
Figure 1: Plane-convex hydrofoil [25]

Figure 2: Computation Domain

Figure 3: Structured and scale distributed mesh with taper analysis of the mesh around the plane-convex hydrofoil 
Figure 4: The Characteristic lengths of a sheet cavity

Figure 5: Comparison of the leading edge cavity growth between numerical simulation result and the experimental result for case (i) in horizontal plane using iso-contour of $\alpha=0.5$

Figure 6: Dimensionless time evolution of cloud shedding for cases from (i) to (v) in 3D using iso-contour of vapor volume fraction $\alpha=0.5$ 
Figure 7: Local pressure coefficient, $C p$, by dimensionless time, $\xi$, for cases from (i) to (v) at different $x / c$. 
Figure 8: Vapor void fraction, $\alpha$, by dimensionless time, $\xi$, for cases from (i) to (v) at different $x / c$.

Figure 9: Comparison between (a) vapor volume fraction isosurface $\alpha=0.5$ and (b) $Q$ criterion vorticity isosurface $Q=2 \times 10^{5}\left[s^{-2}\right]$ at $\xi=0.16$. (c) Vertical plane of the $Q$ criterion. 